\title{
Diffusione del dramma pastorale in Istria
}

\author{
Nives Zudič Antonič
}

Nell'articolo, partendo dall'origine e dalla diffusione del dramma pastorale in Italia, viene presentata la diffusione di questo componimento poetico in Istria. Nell'articolo vengono pertanto presentati alcuni degli autori istriani che si sono cimentati nella stesura di questo tipo di componimento poetico e viene fatta pure l'analisi di alcune delle loro opere più significative. Tra gli autori che vengono presi in esame troviamo: Girolamo Vida, Ottonello de’ Belli e Giovan Battista Brati.

Parole chiave: dramma pastorale, fiaba pastorale, poesia idilliaca, egloga, idillio.

V članku najprej predstavimo izvor in širjenje pastoralne igre v Italiji, v nadaljevanju pa predstavimo širjenje te zvrsti v Istri. V članku predstavljamo tri najpomembnejše istrske pisce pastoralnih iger in nekaj njihovih najvidnejših del, ki smo jih analizirali. Predstavljeni avtorji so: Girolamo Vida, Ottonello de Belli in Giovan Battista Brati.

Ključne besede: pastoralna igra, idilična poezija, ekloga, idila.

D all'idea di ampliare l'egloga dialogata, assai cara ai poeti del secondo Quattrocento, in egloga drammatica, con personaggi in veste di pastori, nacque, e presto entrò in voga negli ambienti colti del Cinquecento, un nuovo genere letterario: il dramma pastorale. Pur avendo origine dai testi degli autori classici come Teocrito e Virgilio, questa composizione si distanzia dalla norma aristotelica e occupa uno spazio intermedio fra la tragedia e la commedia, spesso intrecciando alla comicità della commedia la serietà della tragedia e dando a vicende tristi conclusione lieta. In Italia il dramma (o favola) pastorale fece la sua prima prova ufficiale con il Sacrificio (1554) di Agostino Beccari a Ferrara, ma al livello della vera poesia si elevò soltanto con l'Aminta di Tasso e con il Pastor Fido di Battista Guarini. E proprio sull'esempio di questi due autori e dei loro componimenti poetici che si ispirano pure gli autori di drammi pastorali in Istria.

\section{La nascita della favola pastorale drammatica}

Nell'ambito del rinnovato classicismo rinascimentale e del gusto raffinato e mondano delle corti, accanto al ritorno alle vecchie forme del teatro classico, l'idillio pastorale trova una sua appropriata collocazione, colorandosi di elementi nuovi, rispetto al passato più autobiografici e patetici e richiamandosi all'egloga dialogata e quindi avviandosi a diventare favola pastorale drammatica o dramma pastorale. L'idillio pastorale in volgare si diffuse largamente nel Rinascimento, specialmente dopo l'Arcadia di Sannazzaro. Esso è l'espressione dell'eterno bisogno umano di fuga dalla realtà storica (che è necessariamente lotta, conflitto, e quindi sofferenza) per evadere, almeno idealmente, in un mondo pacifico, sereno, remoto dagli affanni della città. Il so-

\footnotetext{
L'idillio ha una lunga tradizione dal greco-siculo Teocrito alle Bucoliche di Virgilio.
} 
gno, cioè, di una vita felice dei campi e dei pastori, paradiso terrestre al di là della storia, che gli uomini avrebbero perduto per l'invenzione della cosiddetta civiltá. In tal senso la poesia idillica divenne sempre più sinonimo di poesia agreste e pastorale.

Il dramma pastorale rispetta le unità del teatro classico e la sua caratteristica è quella di rappresentare vicende tristi che però si concludono con un lieto fine, soddisfando le esigenze dell'artista rinascimentale, perché la massima aspirazione del poeta di corte cinquecentesco è il sogno di sublimazione e rispecchiamento del proprio travaglio esistenziale, proiettato in un mondo consapevolmente ormai ritenuto fittizio e artificiale, e insieme espressione idealizzata del dramma della passione. Da ciò l'esigenza del travestimento teatrale dei personaggi della corte, in cui gli stessi cortigiani si rappresentano e si contemplano come su un palcoscenico. ${ }^{3}$

Di uno sviluppo drammatico dell'egloga, che diventa sempre più complessa, assumendo motivi della commedia, della tragedia e della farsa, si possono seguire le tracce sin dai primi decenni del Cinquecento; ma di un vero e proprio dramma pastorale non si può parlare prima del I554, quando fu recitato a Ferrara il Sacrificio di Agostino Beccari, ferrarese (I510 c. - I590), in cui le semplici scene pastorali si sviluppano in regolare assetto drammatico e in cinque atti.

In Italia i più importanti drammi pastorali del Cinquecento sono l'Aminta del Tasso e il Pastor fido, del ferrarese Battista Guarini (1538I6I2). L'Aminta è una favola pastorale rappresentata nel luglio del 1573 nell' isola di Belvedere sul Po, davanti alla corte estense.

Gli studiosi hanno segnalato tutte le fonti di questa favola: Teocrito, Mosco, Virgilio, Ovidio, Petrarca, Poliziano, Sannazzaro, ecc. Si tratta quindi di un'opera molto letteraria per il contenuto, ma quello che è più importante è il tono tutto personale con cui la materia è raccontata e liricamente espressa. Inoltre, è rilevante il fatto

2 Mario Sansone, Disegno storico della letteratura italiana (Milano: Principato, 1963).

3 Alberto Asor Rosa, Storia della letteratura italiana (Firenze: La Nuova Italia, 1985). che i personaggi sono un travestimento pastorale di certe figure della corte estense: Licori è Lucrezia Bendidio, Elpino il vecchio innamorato di Licori, è il Pigna, sotto l'aspetto di Batto si nasconde il Guarini, ecc. ${ }^{4}$

L'Aminta è, per così dire, la corte che contempla se stessa staccandosi momentaneamente dalla realtà e rifugiandosi in un sogno di bellezza e di armonia naturale. $\grave{E}$ ancora un prodotto del Rinascimento maturo, che però rivela i segni dell'imminente decadenza. La sensualità, l'erotismo sono al centro della fragile vicenda. Si veda il coro dell'Atto I che celebra l'eden primitivo: "O bella età dell'oro...", in cui il piacere era il massimo fine della vita, e la regola "se piace, ei lice"; ' ma già un velo di malinconia attenua la gioia di vivere e godere, e il dramma manca di azione e si stempera in effusione lirica. ${ }^{6}$

Il Pastor Fido (I590), dramma pastorale o, meglio, tragicommedia come l'autore stesso preferiva chiamarlo, perché si proponeva di fondere gli elementi della tragedia e della commedia classica. Infatti, egli deriva dalla tragedia la statura eroica di certi personaggi e la drammaticità, spesso solo fittizia, dell'intrecciarsi di eventi sorprendenti, e dalla commedia il programmato smorzarsi e il sorridente e garbato sciogliersi delle passioni nella grazia dell'idillio.'

Il suo scopo, come afferma Guarini, non è ammaestrare moraleggiando, ma solo dilettare $\mathrm{i}$ suoi lettori; e siccome non c'è diletto senza sofferenza, come non c'è corpo senza ombra, mescola il tragico e l'elegiaco col comico, per creare tensioni e attesa cui deve seguire appagamento e rilassatezza. ${ }^{8}$

È evidente l'intenzione del Guarini di superare l'Aminta del Tasso, ma l'Aminta è un'opera di maggior respiro sentimentale e unità espressiva e stilistica rispetto a quella del Guarini, dove, tuttavia, per il gusto della magnificenza orna-

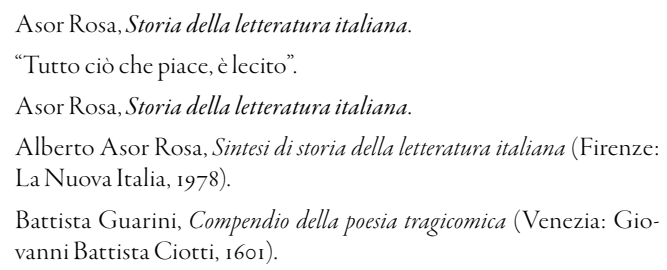


mentale e per il risalto che hanno i momenti lirici su quelli descrittivi, già prelude alla grande poesia e alla musica barocca. Anche l'erotismo e la celebrazione della pura sensualità sono elementi dominanti in questo dramma pastorale che indulge al piacere senza problemi e scrupoli moralistici.?

Va comunque ricordato che sia il Guarini che il Tasso sono uomini del loro tempo: ambedue uomini di corte, e partecipi di quel sogno di fuga dalla rumorosità mondana, nella pace idilliaco-campestre che era un connotato tipico della cultura cortigiana.

\section{L'ispirazione idillica e pastorale dell'Arcadia}

Nell'ottobre del 1690 a Roma, un gruppo di poeti e intellettuali si costituirono in una nuova accademia cui dettero il nome di Arcadia. I maggiori ispiratori e iniziatori di tale movimento, che si richiamava all'antica poesia idillica, bucolica e pastorale, furono Giovan Mario Crescimbeni e Gian Vincenzo Gravina.

I poeti aderenti all'Arcadia predilessero, in opposizione al gusto barocco ancora dominante, un linguaggio d'ispirazione classicistica volutamente semplice e lineare, adatto al genere della poesia bucolica, in cui cortigiani e dame (come nell'Aminta tassiana) travestiti in pastori, pastorelle e ninfe si muovevano su uno sfondo campestre sereno, tipico della tradizione idillica, simboleggiante l'antico sogno di fuga dalla triste realtà della vita. ${ }^{10}$

Acutamente il Gravina definì l'Arcadia: "un sogno in vista della ragione", riassumendo abilmente in una sigla la matrice razionalistica (ma di stampo moderato) di questa poetica.

Immagini campestri molto convenzionali costituiscono il palcoscenico su cui si atteggiano in pose languide e sospirose amanti e amate fra parrucche e trine che, nei momenti poetici più fortunati, sospendono in una nube soffusa di ciprie e profumi i poetici segni di una società

9 Natalino Sapegno, Compendio distoria della letteratura italiana $(\mathrm{Fi}-$ renze: La Nuova Italia, 1951).

Io Asor Rosa, Storia della letteratura italiana. elegante e colta, sensibile alle galanterie e svenevolezze studiate dell'ambiente cortigiano e curiale. $^{\text {II }}$

Il gusto cui abbiamo accennato del travestimento, induce i poeti a darsi degli pseudonimi idillico-pastorali. Le donne amate, coerentemente, si chiamano Filli, Nice, Nerina, nome che incontriamo, p. es., in queste strofe di Paolo Rolli (da L'Inverno, quinto libro delle sue Rime): ${ }^{\mathrm{I2}}$

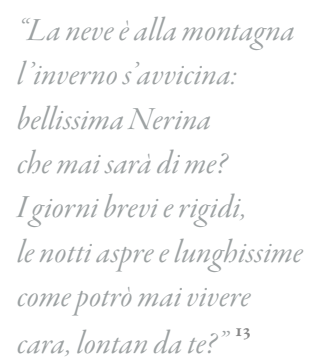

La semplicità proposta dagli Arcadi è certamente raggiunta in questi versi. La successione di settenari intreccia abilmente quella musica insieme saltellante e sospirosa che si ritroverà nel Metastasio, e che è connotato saliente caratteristico dei poeti arcadici, fra i quali Carlo Innocenzo Frugoni, Giovan Battista Zappi, Aurelio Bertola. Dello Zappi c'è da dire che l'eccesso di dolcezza delle sue liriche gli meritò l'attributo datogli dal Baretti di inzuccheratissimo. ${ }^{14}$ Ed è questo, infatti, il limite della poetica e del linguaggio arcadici, anche se in essi è tuttavia riconoscibile spesso uno schietto amore per la Natura e l'aspirazione a uno stile di vita sereno e remoto dalle convenzioni tormentose della civiltà urbana.

\section{Il dramma pastorale in Istria}

In Istria il dramma pastorale è legato per lo più alle Accademie, che furono un segno distintivo dell'età rinascimentale. Esse comparvero dapprima come semplici associazioni erudite, per trasformarsi successivamente in un sodalizio dotato di norme e leggi particolari. La caratteristica

\footnotetext{
I I Asor Rosa, Storia della letteratura italiana..

I2 Quello delle stagioni fu un genere diffuso nel Settecento.

I3 Paolo Rolli, Dépoetici componimenti del Signor Paolo Rolli: divisi in trelibri (Venezia: Bartolommeo Occhi, 176I), 229.

14 Asor Rosa, Storia della letteratura italiana.
} 
che accomunava questi circoli di dotti era lo studio delle discipline scientifiche e letterarie. La loro diffusione fu una conseguenza del risveglio culturale e della rinnovata vita intellettuale e sociale.

A Capodistria, principale centro urbano dell'Istria, che annoverava le più importanti istituzioni culturali, le accademie comparvero nell'ultimo quarto del XV secolo, proprio come nel resto d'Italia. Dal I 478 al 1567 era attiva la Compagnia della Calza (all' inizio prevalsero gli esercizi cavallereschi ma si trasformò poi in sodalizio letterario). Importante anche l'Accademia dei Desiosi, sorta nel 1553 per iniziativa di un gruppo di intellettuali, si sciolse l'anno successivo perché sospettata di eresia da Girolamo $\mathrm{Mu}-$ zio. Nel 1554 essa si tramutò in Accademia Palladiana o dei Palladi (fu chiusa nel 1637) e ebbe tra i suoi aderenti: Santorio Santorio, che ne fu per qualche tempo "principe"; Girolamo Vida, Ottonello de Belli, Guido de Belli, Giacomo Zarotti, Annibale Grisonio, Mario Vida, Nicolò Manzuoli. ${ }^{15}$ I membri che ne facevano parte, seguendo il genere allora in voga, proponevano perlopiù drammi pastorali. Pietro Pola presentò l'Ardor di Amore e la commedia I giusti inganni, Girolamo Vida la Filliria e il dialogo Il Sileno, Ottonello de' Belli Le selve incoronate e il poemetto satirico Lo scolare, Giovan Battista Brati scrisse il dramma pastorale La ninfa del Formio$n e^{16}$

Nel 1646 fu fondata l'Accademia dei Risor$t i$ che con alterne vicende rimase in vita sino al I806. Vi facevano parte: Giuseppe e Cristoforo Gravisi, Domenico Manzioli, Antonio Grisoni, Giacomo de Belli, Gavardo Gavardo, Cristoforo Tarsia, Giuseppe Bonzio, Moretti e Alvise Manzioli. Questo sodalizio accolse anche il medico Girolamo Vergerio, poi professore nelle università di Pisa e Padova e Cesare Zarotti, medico, poeta, epigrammista. Nel 1739 al suo interno vi fu una scissione promossa da Girolamo Gravisi e dal cugino Gian Rinaldo Carli e fu fondata l' $A c$ -

\footnotetext{
I5 Baccio Ziliotto, Accademie e accademici di Capodistria (1478-1807) (Trieste: Arti grafiche L. Smolars \& nipote, 1944).

I6 Nives Zudič Antonič, Storia e antologia di Capodistria, Isola e Pirano (Capodistria: Unione Italiana, 20 I4)
}

cademia degli Operosi (1739-1742). Essa desiderava dare un contributo nuovo e concentrò l'attenzione soprattutto sugli studi di storia antica, ma ebbe vita breve poiché i suoi giovani membri lasciarono la città per frequentare l'università a $\mathrm{Pa}$ dova, compresi i due promotori (Carli nel 1740 fu accolto nell'Accademia dei Ricovrati della città veneta). Fu rifondata nel 1763 come una sorta di cenacolo privato di giovani poeti; ne fu promotore Dionisio Gravisi e si estinse con la sua prematura morte (I767). ${ }^{17}$

\section{Gli autori istriani di drammi pastorali e poesie di ambientazione agreste}

Come abbiamo detto nel paragrafo precedente, un aspetto particolare della letteratura istriana del Cinquecento e del Seicento è costituita dalle Accademie, che si collegano per più riguardi al movimento umanistico, ma che hanno una loro specifica autonomia e uniscono a una componente culturale una componente salottiera e mondana, apparendo espressione caratteristica della società nobiliare e, comunque, di una cultura elitaria e aristocratica. Proprio in questi luoghi pertanto gli intellettuali amavano discutere di letteratura e presentare le proprie opere.

Nel Cinquecento uno dei primi autori di un'egloga pastorale fu il capodistriano Pietro Pola, autore di diverse commedie e di un'egloga pastorale. Girolamo Vida nel 1585 scrive la favola pastorale o boschereccia Filliria che racconta la storia di Nelirio innamorato di Filliria, una ninfa che gli sfugge. L'opera venne rappresentata per la prima volta dall'autore a Capodistria il 27 gennaio del I585; il cav. march. Giovanni Niccolò Gravisi di Capodistria creò le scene per lo spettacolo. ${ }^{18}$ Nello stesso anno l'opera fu rappresentata a Padova, di seguito a Venezia nel 1587 e nuovamente a Padova nel i621. Per quest'opera, Girolamo Vida fu eletto membro dell'Accademia Olimpica di Vicenza.

Il dramma pastorale è un componimento poetico spesso usato dagli autori italiani. Da un

\footnotetext{
I7 Baccio Ziliotti, Storia letteraria di Trieste e dell'Istria (Trieste: La Editoriale Libraria, 1924)

I8 Nives Zudič Antonič, Storia e antologia di Capodistria, Isola e Pirano.
} 
punto di vista retorico, ciò che accomuna questi drammi è la strutturazione in cinque atti e l'osservazione delle unità di tempo, luogo e azione; lo scenario prescelto è quello dell'Arcadia o della Grecia antica o dell'Italia (nel nostro caso anche quello dell'Istria), quasi tutti sono in versi endecasillabi. Un altro elemento distintivo è la struttura dell' intrigo, uno schema complesso, con un notevole numero di personaggi solitamente presentati in coppie, in cui casi amorosi si intrecciano l'un l'altro. Gli autori istriani per scrivere le loro opere si rifanno ai lavori dei grandi scrittori italiani, di cui sono grandi ammiratori. A tal proposito lo scrittore inglese Richard Hughues che nel 1927 soggiornò a Capodistria a proposito dei drammi pastorali istriani del Cinquecento disse: "Nulla di originale; in tutta l'Europa nel Cinquecento si cantavano satiri e ninfe, ma se tutta la poesia istriana di quel secolo è così, parlerei d'imitazione di opere classiche [...]."'

Altro autore capodistriano di drammi pastorali è Ottonello de' Belli che compose il dramma pastorale Le selve incoronate (pubblicate dagli eredi dopo la sua morte, nel 1673 a Venezia). Le selve incoronate è una "tragicommedia", cioè un misto di tragedia e commedia che si rifà al dramma pastorale Pastor Fido del Guarini. L'opera mantiene l'unità di tempo e di luogo ed è composta di cinque atti.

L'autore, come il Guarini, fonde nella sua tragicommedia elementi della tragedia e della commedia classica. Egli deriva dalla tragedia la statura "eroica" di certi personaggi e la drammaticità, spesso solo fittizia, dell'intrecciarsi di eventi sorprendenti, e dalla commedia il programmato smorzarsi e il sorridente e garbato sciogliersi delle passioni nella grazia dell'idillio.

Giovan Battista Brati è autore del dramma pastorale La ninfa del Formione (1617), ${ }^{2 \circ}$ che venne considerata la sua opera migliore, anche se $\mathrm{i}$ critici esprimono giudizi poco favorevoli. La fiaba narra la storia di Mirzio pastore innamorato

I9 Francesco Semi. Istria e Dalmazia: uomini e tempi. Istria e Fiume, 2 I 5.

20 Formione: antico nome del fiume Risano di Lidia, una fanciulla che rifiuta il suo amore, ${ }^{21}$ per farle cambiare idea Mirzio decide di raccontarle la tragica storia della ninfa Dori. La bellissima ninfa Dori abitava sulle sponde del fiume Formione.

\section{Diquesto ameno, egrato, \\ lusinghiero soggiorno, \\ fortunato girdin, stanza amorosa. \\ Stanza ben certo degna \\ De'Semidei gentili, \\ $V$ 'era Vergine bella, \\ Nobilposseditrice: \\ DORIgentil nomata, \\ Che qualdal'oro hà il nome: \\ Cosid'oro ha le chiome \\ Eralagiovinetta \\ De la bella età sua su quel confine, \\ Che pius s'appressa all'acerbetto Aprile; \\ Bella sine' anni, \\ Ch'Angioletta sembrava in forma humana \\ Scesa dal Ciel qua in terra, \\ Perfar del Cielo amato \\ Disuebellezze, innamorar la Terra.}

Nel suo giardino, che si trovava lungo le rive del fiume, la ninfa Dori coltivava dei bellissimi fiori, inoltre pescava nel fiume, nelle vicinanze di quelle sponde andava a caccia e non si curava dell'amore.

\section{Etalhora colveltro \\ Seguir difera fuggitival'ormè, \\ Ospiando il covile \\ De le timide lepri, e de conigli, \\ Òcon la canna, el'hamo \\ Turbar nel' acqua a' pesciriposo. \\ Òco'l visco, ò co'llaccio, \\ Tender à vaghi augelli insidie ascose. ${ }^{23}$}

Il pastore Eurindo era innamorato di Dori e un mattino, mentre lei pescava, le si avvicinò e le dichiarò il suo amore. Ma Dori, che aveva dedi-

\footnotetext{
21 Il tema della resistenza all'a more è un tópos della letteratura idillica. Nell'Aminta del Tasso, p. e. è la ninfa Silvia che si vorrebbe sottrare all'amore, che è non solo legge di Natura, ma anche forza d'ingentilimento'.

22 Giovan Battista Brati, La ninfa del Formione (Venezia: 1619), 5-6.

23 Giovan Battista Brati, La ninfa del Formione, 7-8.
} 
cato a Diana la propria verginità, pregò la dea di difenderla dal giovane. Diana la trasformò in acqua, mentre Zeffiro col suo dolce vento, e Apollo con la sua potenza, agitarono le onde del Formione: e le piccole stille "mirabilmente fersi/ picciol globi di sale".

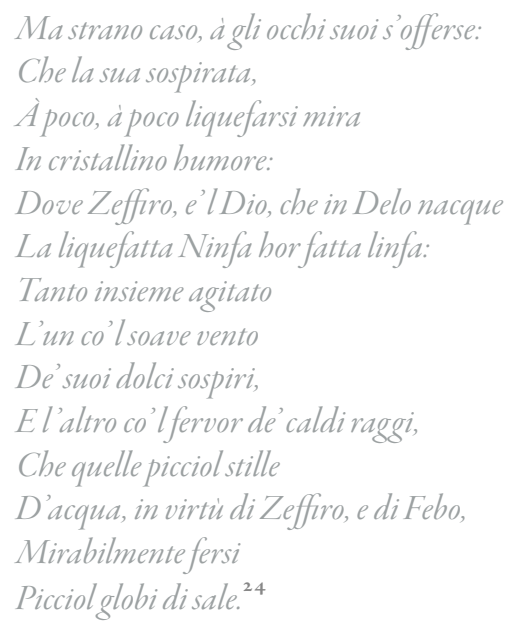

Eurindo allora, aiutato da Nereidi, raccolse quei granelli che da quel giorno divennero condimento del cibo. Alla fine del racconto di Mirzio scende la sera e così si conclude pure la fiaba del Brati.

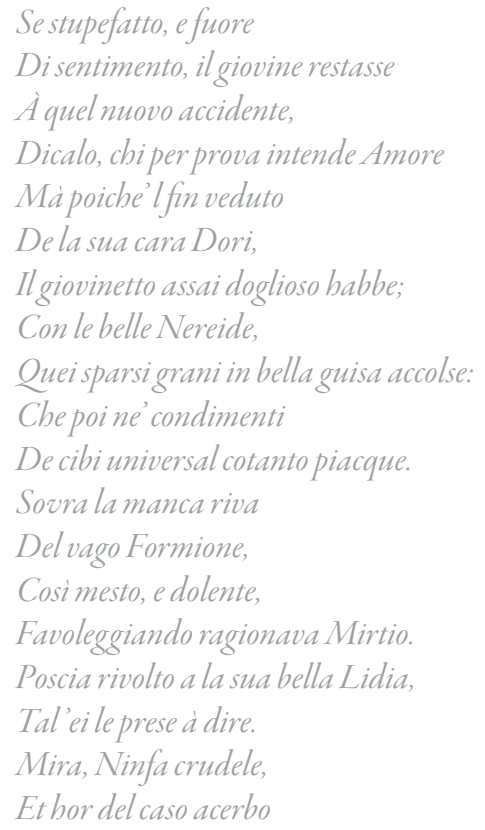

Di Dori, impara a le sue spese, o bella,

A non insuperbire,

Con tra Amor; tu che troppo

Di tua beltà sai de la schiva, tale

De le donne superbe è la mercede:

Tu gradisci il mio affetto, e mentre il giorno

Fè con la notte tregua,

Silentio imporse al ragionar', e tacque.

Nel dramma pastorale il poeta oltre a raccontare la storia dei due innamorati presenta una descrizione molto accurata del paesaggio primaverile lungo il corso del fiume Formione (odierno Risano) e rifacendosi all'esempio della ballata di Angelo Poliziano I'mi trovai, fanciulle presenta tutti i fiori che ci sono nei prati che si trovano lungo il corso del fiume. Anche qui però come nella ballata di Poliziano la rosa viene presentata come la regina di tutti i fiori.

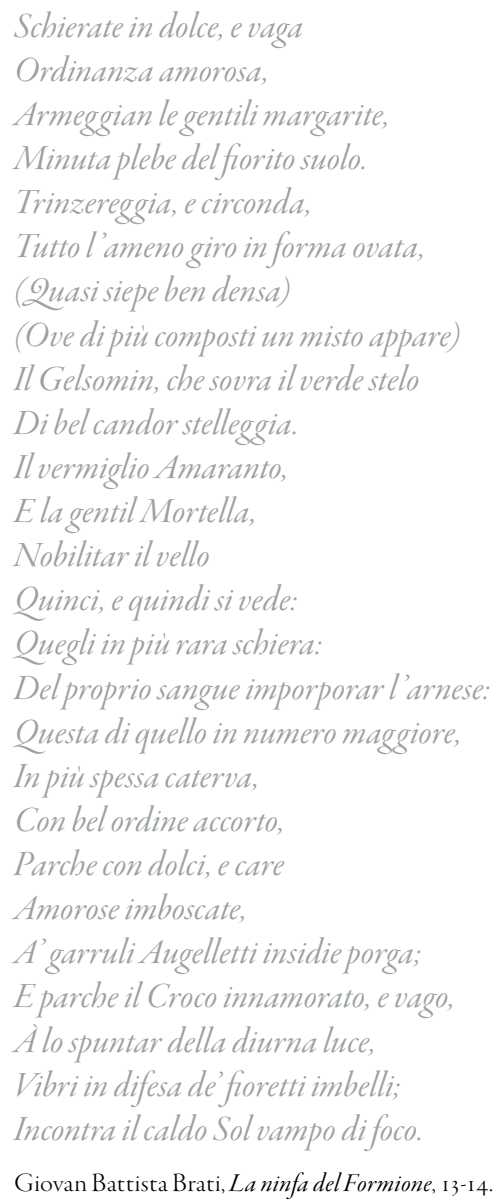

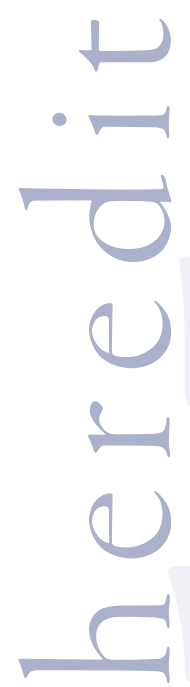




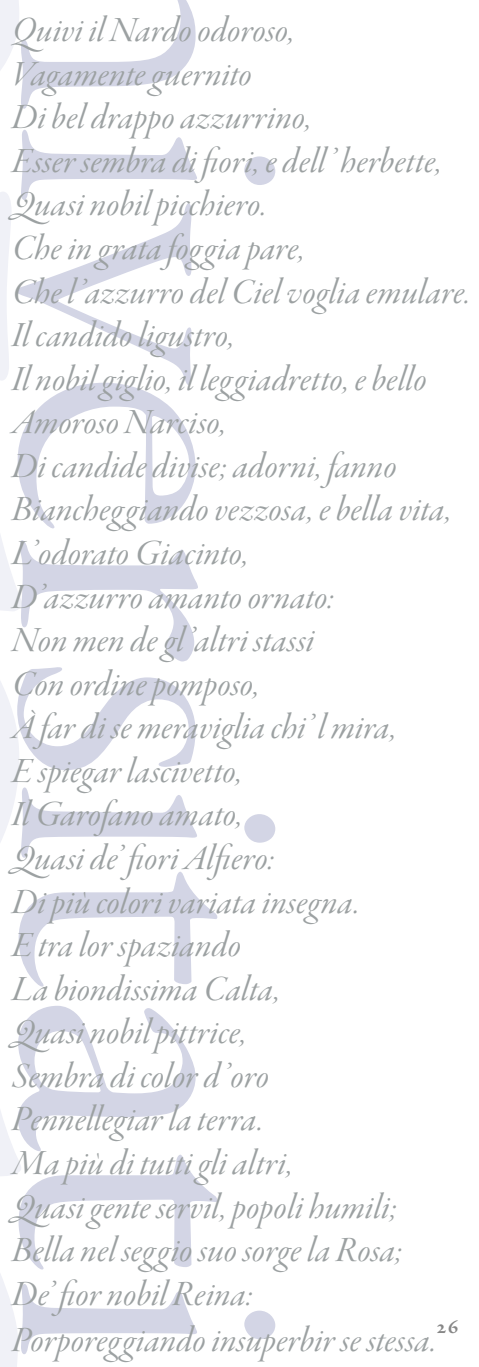

Il motivo dei fiori e in particolar misura della rosa ha numerosi riscontri nella produzione degli autori dei drammi pastorali in Istria. Spontaneo è il rimando alla ballata del Poliziano: comune ai due testi è il tema della poesia d'amore che si confonde con la natura fiorita, il rilievo dato alle notazioni cromatiche (i versi presentati sopra rappresentano un autentico catalogo dei colori del prato), l'importanza di trovarsi in un paesaggio primaverile fiorito.

Mentre però il Poliziano nella sua ballata parla della raccolta dei fiori da parte della donna per farne una ghirlanda che l'avrebbe poi adornata, Brati si rifà alla descrizione dei fiori fino ad arrivare alla rosa come regina tra tutti per presentare l'ambiente in cui vive la protagonista del suo dramma. Per queste opere gli autori si rifanno a due modelli classici: un testo greco del retore Libanio, in cui Venere, in riva al fiume Scamandro, sceglie fra tutti i fiori le rose, con cui si adorna prima del giudizio di Pride; e un anonimo carme latino De rosis. Va inoltre ricordato che il Poliziano dedica il capitolo XI della prima centuria dei suoi Miscellanea al motivo di Venere e della rosa, ripercorrendo diverse attestazioni classiche, latine e greche (compreso lo stesso Libanio) inerenti alla tradizione che lega questo fiore all'amore. ${ }^{2}$

Al di là delle fonti e dell' ispirazione, l'originalità dell'opera di Brati sta proprio nella descrizione così minuziosa del paesaggio lungo le rive del Risano e della descrizione leggendaria dell'origine del sale, elemento tanto prezioso per la sua regione.

Nel Settecento in Istria la tradizione dei drammi pastorali viene ripresa da alcuni autori attraverso la composizione di egloghe pastorali o composizioni poetiche che si rifanno all'idillio. Vanno qui ricordati Girolamo Gravisi e suo figlio Dionisio e Giuseppe Bonzio. Giuseppe Bonzio poeta capodistriano, fece parte dell'Accademia dei Risorti tra il 1754 e il 1755 (il suo nome accademico fu Tirsi). Il poeta scrive un libro di poesie assieme a Dionisio Gravisi Poesie liriche che verrà pubblicato dopo la sua morte nel I77I dalla contessa Santa Borisi Gavardo. Nella prefazione al libro, la donna, parlando dei versi del Bonzio dice che "questi si sollevano dalla bassa nojosa turba dei vuoti verseggiatori..." si nota inoltre nei suoi versi una somiglianza ai versi del Chiabrera che fu promotore dell'indirizzo dell'Arcadia classicheggiante e imitò la vacua grandiosità dell'ode pindarica. ${ }^{29}$ La contes-

Riccardo Bruscagli, Gino Tellini Vittorio Corsano Lucia Denarosi e Silvia Fiaschi, Itinerari dell 'invenzione 2. Umanesimo e Rinascimento. Autori, temi, crocevia della letteratura italiana ed europea (Firenze: Sansoni per la scuola, 2002).

28 Santa Borisi Gavardo, "Prefazione," in Poesie liriche dei signori Giuseppe Bonzio e marchese Dionisio Gravisi nobiligiustinopolitani. Giuseppe Bonzio e Dionisio Gravisi, (Venezia, 1771), 3.

29 Nives Zudič Antonič, Storia e antologia di Capodistria, Isola e Pirano. 
sa inoltre spiega che il Bonzio fu imitatore pure di Benedetto Menzini che fu, come specifica lei "uno dei primi, che alle itale muse abbia restituito l'antico decoro oscurato ed offeso dai falsi vezzi dello scorso secolo, in cui egli visse". ${ }^{\circ} \mathrm{La}$ donna dice inoltre che le poesie del Bonzio sono talmente ben scritte e "animate da fuoco poetico" che favoriscono la lettura.

Gli intellettuali che frequentavano l'Accademia dei Risorti solitamente amavano proporre dei problemi su cui discutere e ragionare. Nell'Egloga pastorale tratta dall'opera Poesie liriche Giuseppe Bonzio presenta un'opera scritta assieme a Girolamo Gravisi su imitazione di Virgilio. Il testo presenta un dialogo in cui Alindo (Girolamo Gravisi) e Tirsi (Giuseppe Bonzio) si pongono il problema se sia più vantaggioso avere un animo guerriero o pacifico.

Dionisio Gravisi dal 1763 , quando era studente nel collegio dei nobili di Capodistria, fu uno dei rinnovatori dell'Accademia degli Operosi, fondata da suo padre trent'anni prima. Già all'età di sedici anni scriveva poesie e collaborava alle Accademie locali. Le sue poesie in linea di massima rispecchiavano la malinconia della sua sofferente giovinezza e il presentimento della morte prematura. ${ }^{3 \mathrm{I}}$ Le sue poesie, come già menzionato sopra, vennero pubblicate assieme a quelle del poeta capodistriano Giuseppe Bonzio dalla contessa Santa Borisi Gavardo. La contessa, nella prefazione al libro di poesie, parlando del poeta, dirà che Dionisio Gravisi è "fortunato seguace della lirica scuola aperta in Grecia, e fatta rinascere in Roma da Orazio nell'aureo secolo di Augusto", ${ }^{2}$ pure lui come il Bonzio sono seguaci del Chiabrera e dell'indirizzo dell'Arcadia classicheggiante. La contessa Borisi Gavardo inoltre lo considera seguace pure del poeta Frugoni e lo Stancovich, nella sua Bibliografia degli uomini illustri dell'Istria, dirà che "le poesie del marchese Dioniso piacciono, allettano, e ricolmano l'animo di un pieno, a cui nulla più resta da desiderare" (1829).

\footnotetext{
30 Gavardo, "Prefazione."

3 I Baccio Ziliotti, Storia letteraria di Trieste e dell'Istria.

32 Gavardo, "Prefazione," 3.
}

Nella poesia L'Armonia il poeta si rifà al componimento pastorale che è l'indirizzo tipico della poesia arcadica. Questo mondo arcadico, spesso così ricco di grazia e di delicatezza fine e superficiale, trovò il suo modo proprio di espressione nella canzonetta in cui si continua l'anacreontica chiabresca, che è propria gloria e, nella sua particolare intonazione, vera creazione d'Arcadia. $^{33}$

La canzone L'Armonia di Dionisio Gravisi, composta da strofe con ritmo facile, e disposta ad accogliere il rivestimento musicale, è un componimento poetico di grazia, di psicologia amorosa facile e squisita insieme. In questo testo si possono intravedere le caratteristiche classicheggianti del Chiabrera.
[...] In mezzo a cuiverdeggerà sublime
L'alma fronde di Pallade Risorta ${ }^{34}$
Che rediviva di nascenti germi
Tanta ora in Pindo di sè parte ingombra.
Mobil dai rami penderan le cetre
Che faran dolce ai venticelli invito,
E desteranno, se movendo, un suono
Di concorde armonia; là presso cinto
Di musco, e canne, il Formion divise
Verserà in rami dalla facil' urna
L'acque cui lungo a fabbricar lor nido
Verran candidi Cigni, aerea Torma,
E un nuovo canto tempreranno, un nuovo
Meonio Fonte risonar faranno. ${ }^{35}$

Nell'altra canzonetta Alla barchetta, che 1 conduce Egle alla campagna Dionisio Gravisi descrive il viaggio in barca che porta Egle $^{36} \mathrm{da} \mathrm{Ca-}$ podistria alla contrada campestre di Arzioli dove la famiglia del poeta aveva una villa.

\footnotetext{
33 Bruno Maier, Letteratura italiana dell' Istria dalle origini al Novecento (Trieste: Edizioni Italo Svevo, 1996).

34 Pianta di Olivo rinascente, esposta al Sole, col motto di far rivivere (rediviva) l'impresa dell'antica Accademia dei Risorti a Capodistria.

35 Giuseppe Bonzio e Dionisio Gravisi, Poesie liriche dei signori Giuseppe Bonzio e marchese Dionisio Gravisi nobili giustinopolitani (Venezia, 1771), 236-237.

36 Egle è il nome accademico di Dionisio Gravisi.
} 
] O tu Risan, che moder

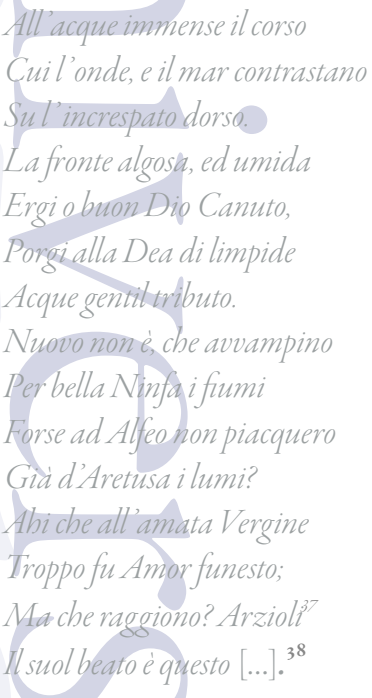

In questa canzone il poeta si rifà al gusto della finzione pastorale e alla trasfigurazione fiabesca del paesaggio. I versi del Gravisi riportano a un senso di misura che si oppone agli eccessi dello stile barocco, puntando piuttosto alla raffinatezza e alla leggiadria della forma. ${ }^{39}$ Nei suoi versi, pertanto, possiamo intravedere il gusto barocco classicheggiante della poesia chiabresca.

Come il Chiabrera anche il Gravisi fece uso di soggetti tratti dai classici greco-ellenistici usando un sistema metrico-linguistico che gli consentì una musicalità chiara e leggera del verso, portando la sua canzonetta a quella caratteristica votata al grazioso.

\section{Conclusione}

Nonostante i critici esprimessero opinioni poco lusinghiere nei confronti degli istriani autori di drammi pastorali, sottolineando che, in genere, detti autori proponevano opere dalla scarsa originalità e di imitazione, va tuttavia considerato che la loro presenza è un'importante testimonianza per la letteratura della regione istriana.

37 Arzioli, contrada campestre, disgiunta per breve tratto di mare da Capodistria, dove c'era una villa dell'Autore.

38 Giuseppe Bonzio e Dionisio Gravisi, Poesie liriche dei signori Giuseppe Bonzio e marchese Dionisio Gravisi nobili giustinopolitani, 236-237.

39 Bruno Maier, Letteratura italiana dell'Istria dalle origini al Novecento.
Ne sono dimostrazione opere quali Filliria, di Girolamo Visa, Le selve incoronate, di Ottonello de' Belli, La ninfa del Formione, di Giovan Battista Brati, che, pur essendo imitazioni delle opere di importanti letterati italiani autori di drammi pastorali, quali Guarini e Tasso, presentano un importante elemento originale riscontrabile nella descrizione dell'ambiente. Quest'ultimo, infatti, è un ambiente locale che, pur rifacendosi alle descrizioni dei grandi autori del Rinascimento italiano, mantiene le caratteristiche e le peculiarità della regione istriana.

\section{Povzetek}

Nova literarna zvrst pastoralne lirike se je razvila iz ideje, da bi razširili eklogo v obliki dialoga, ki je bila posebno pri srcu pesnikom petnajstega stoletja, $\mathrm{v}$ dramatično eklogo s pevci v vlogi pastirjev: nova literarna zvrst je kmalu postala zelo popularna in se razširila v kulturnih krogih šestnajstega stoletja. Čeprav je njej nastanek vezan na klasične avtorje, predvsem na Teokrita in Vergilija, pesniki pastoralne lirike ne spoštujejo aristotelskih norm, ker združujejo bodisi nekatere značilnosti tragedije kot komedije, saj večkrat začinijo resnobo tragičnih dogodkov s komičnim humorjem ali pa pripišejo srečen konec žalostnim dogodkom.

V Italiji je bila pastoralna lirika prvič predstavljena v Ferrari z delom Sacrificio (1554) Agostina Beccarija, svoj višek žlahtne poezije pa je doseglas Tassejevo Aminto in z delom Battista Guarinija Pastor Fido. In ravno oba omenjena pesnika in njuni pesnitvi sta navdihnila pesnike pastoralne lirike v Istri. V Istri je bila pastoralna lirika še najbolj prisotna v Akademijah, ki so imela pomembno vlogo v času renesanse. Ta središča, ki so bila sicer bolj povezana s humanističnim gibanjem, so pa imela specifično avtonomijo, so prepletala neresne in posvetne tematike s kulturnimi kot karakteristična značilnost aristokratske družbe in, elitne, aristokratske kulture. Ravno v teh krogih so istrski intelektualci radi razpravljali o literaturi in predstavljali svoja dela.

Med avtorji, ki so opisani v članku gre omeniti: Koprčana Pietra Polo, ki je bil prvi avtor pastirske ekloge v šestnajstem stoletju; Girolamo Vida je leta I586 napisal Fillirio, pastoralno zgodbo ali boschereccio; leta 1673 je bila objavljena pastoralna drama Le selve incoronate Koprčana Ottonella de’ Bellija; leta I6ı7 je Giovan Battista Bra- 
ti napisal pastoralno dramo La ninfa del Formione. Članek omenja še druge pisce, kot so bili Girolamo Gravisi, njegov sin Dionisio, in Giuseppe Bonzio, ki je nadaljeval tradicijo pastoralne drame v osemnajstem stoletju v Istri s pastoralnimi eklogami ali pesniškimi kompozicijami, povezanimi zidilami.

Ključne besede: pastoralna igra, idilična poezija, ekloga, idila

\section{Summary}

From the idea of expanding an eclogue in dialogues, very dear to the poets of the second half of the fifteenth century, into a dramatic eclogue, with characters in the roles of shepherds, a new literary genre was born which soon became popular among the educated circles of the sixteenth century: pastoral drama. Although originating from the texts of Classical authors such as Theocritus and Virgil, this form does not conform to the Aristotelian norm, and occupies an intermediate space between tragedy and comedy, often interweaving the seriousness of tragedy with the humour of comedy, and giving unhappy events a happy ending. In Italy, pastoral drama (or fable) made its first appearance in Ferrarra with Sacrificio (1554) by Agostino Beccari; however, it rose to the level of genuine poetry only with the Aminta by Tasso, and with the Pastor Fido by Battista Guarini. In fact, it is the example of these two authors and their poetic compositions that also inspired the authors of pastoral drama in Istria. In Istria, pastoral drama is mostly related to the Academies, which were a distinctive feature of the Renaissance period. These centres, which were connected more to the humanistic movement, but have a specific autonomy, combine a frivolous and worldly component with a cultural one, appearing as a characteristic expression of aristocratic society and, of an elite and aristocratic culture. It is precisely in these places, therefore, that Istrian intellectuals enjoyed discussing literature and presenting their works.

Among the authors presented in the article are: the Capodistrian, Pietro Pola, who was the first author of a pastoral eclogue in the sixteenth century; in ${ }^{5} 85$ Girolamo Vida, wrote Filliria, a pastoral fable or boschereccia; in 1673 the pastoral drama Le selve incoronate by the Capodistrian Ottonello de'Belli was published; in 1617 Giovan Battista Brati wrote the pastoral drama La ninfa del Formione. The article also cites some authors, such as Gi- rolamo Gravisi, his son Dionisio, and Giuseppe Bonzio, who continued the tradition of pastoral drama in the eighteenth century in Istria by writing pastoral eclogues or poetic compositions that relate to idyll.

Keywords: pastoral drama, pastoral fable, idyllic poetry, eclogue, idyll.

\section{Bibliografia}

Asor Rosa, Alberto. Sintesi di storia della letteratura italiana. Firenze: La Nuova Italia, 1978 .

Asor Rosa, Alberto. Storia della letteratura italiana. Firenze: La Nuova Italia, 1985.

Borisi Gavardo, Santa. "Prefazione.” In Poesie liriche dei signori Giuseppe Bonzio e marchese Dionisio Gravisi nobili giustinopolitani, Giuseppe Bonzio e Dionisio Gravisi, 2-5. Venezia, I77I.

Bonzio, Giuseppe, e Dionisio Gravisi. Poesie liriche dei signori Giuseppe Bonzio e marchese Dionisio Gravisi nobili giustinopolitani. Venezia, I77I.
$\downarrow$
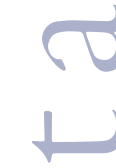

Brati, Giovan Battista. La ninfa del Formione. Venezia, I6I9.

Bruscagli, Riccardo, Gino Tellini, Vittorio Corsano, Lucia Denarosi, e Silvia Fiaschi. Itinerari dell'invenzione 2. Umanesimo e Rinascimento. Autori, temi, crocevia della letteratura italiana ed europea. Firenze: Sansoni per la scuola, 2002.

De'Belli, Ottonello. Le selve incoronate. Tragicomedia Boschereccia del signor D. Ottonello de Belli nobile giustinopolitano. Venezia, 1673.

Guarini, Battista. Compendio della poesia tragicomica. Venezia: Giovanni Battista Ciotti, I6or.

Maier, Bruno. Letteratura italiana dell'Istria dalle origini al Novecento. Trieste: Edizioni Italo Svevo, 1996.

Rolli, Paolo. De’ poetici componimenti del Signor Paolo Rolli: divisi in tre libri. Venezia: Bartolommeo Occhi, I77I.

Sansone, Mario. Disegno storico della letteratura italiana. Milano: Principato, 1963. 
Sapegno, Natalino. Compendio di storia della letteratura italiana. Firenze: La Nuova Italia, I95I.

Semi, Francesco. Istria e Dalmazia: uomini e tempi. Istria e Fiume. Udine: Del Bianco Editore, 1991.

Stancovich, Pietro. Biografia degli uomini distinti dell'Istria del canonico Pietro Stancovich. Tomo secondo. Trieste: Presso Gio. Marenigh Tipografia, I829.

Ziliotti, Baccio. Storia letteraria di Trieste e dell'Istria. Trieste: La Editoriale Libraria, 1924.

Ziliotto, Baccio. Accademie e accademici di Capodistria (1478-1807). Trieste: Arti grafiche L. Smolars \& nipote, 1944.

Zudič Antonič, Nives. Storia e antologia di Capodistria, Isola e Pirano. Capodistria: Unione Italiana, 2014.

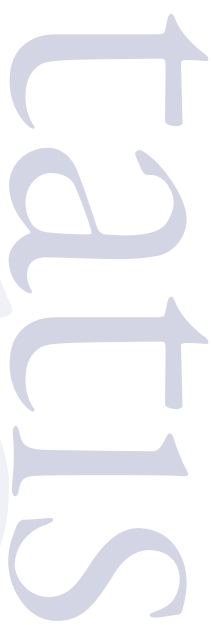

